\title{
Study on Professional Commitments of Nursing Students After Completing Their Final Clinical Electives
}

\author{
Shu-Chen Wang ${ }^{1}$ and Yu-Hsiu Kao ${ }^{2 *}$ \\ ${ }^{1}$ Internship instructor, Department of Nursing, Asia University \\ ${ }^{2}$ Associate Professor, Graduate of Institute of Health Allied Education, National Taipei University of Nursing Health Sciences
}

*Corresponding author: Yu-Hsiu Kao, Associate Professor, Graduate of Institute of Health Allied Education, National

Taipei University of Nursing Health Sciences, Taiwan

ARTICLE INFO

Received: 蔧 September 09, 2020

Published: 蔧 September 17, 2020

Citation: Shu-Chen Wang, Yu-Hsiu Kao. Study on Professional Commitments of Nursing Students After Completing Their Final Clinical Electives. Biomed J Sci \& Tech Res 30(3)-2020. BJSTR. MS.ID.004966.

Keywords: Nursing Students;Final Clinical Electives; Nursing Professional Commitment

\section{ABSTRACT}

Research Goals: To understand the professional commitments of nursing students after completing their final clinical electives and to explore the different personal background factors affecting these commitments in the nursing profession.

Research Methods: This study made use of a cross-sectional questionnaire, whichused the scale for nursing professional commitment developed by Lü (2001), for which a letter of consent was obtained from the author. The research subjects were nursing students at northern Taiwanese university who had completed their final clinical elective courses. 389 valid questionnaires were collected.

\section{Results}

(1) The four dimensions of "professional commitment" are generally ranked from high to low as recognition of value, willingness for career involvement, positive career assessment, and willingness to exert effort.

(2) The score for overall professional commitment of nursing students was relatively high for those whose responses included "personal interest" and "easy employment" ,"two skills", "no support," and "some support.." This was also true for those who rated their health as "excellent" and "very good," whose scores were "above 85\%,", whose elective hospital level was "medical center,", and for whose medical department was "internal medicine,", "surgery and operating room" and "emergency and intensive care".

\section{Recommendations}

(1) Helping nursing students during their studies has a positive impact on their educational success, which in turn can raise the level of professional commitment of these students.

(2) In their final clinical elective units, nursing students should be provided with greater opportunities to strengthen their working abilities; this will help improve their self-confidence in their nursing work.

\section{Introduction}

Although there is sufficient cultivation of Taiwanese nursing manpower, but problems of chronic shortage of nursing manpower, new nurses, and job changes for medical institutions $\mathrm{Hu}$, et al. [1], freshly graduated nurses must face the shortage of nursing manpower, and complicated work challenges immediately thrust upon them upon entering their profession.Strengthening the practical nursing experience that students receive in school, thus allowing them to recognize the urgency of their own profession, 
is crucial in helping new nurses to adapt to their new work environment, and to build their professional work skills $\mathrm{Wu}$, et al. [2]. The development of commitment is an internalized process, and is thus influenced by factors such as internal personal characteristics, family background, and environment Lü, et al. [3]. Professional commitment includes "professional dedication" and "organizational commitment,," and can be an important predictive indicator of willingness to work and level of involvement Benligiray, et al. [4]. If we are able to understand the factors related to the final clinical electives of nursing students which affect their professional commitment, then it will be possible to help administrators for schools, professors, and the industry to arouse a sense of professional mission in nursing students in their design of these final clinical electives.

Although many researchers discuss in detail the phenomenon of the cultivation of professional commitment during the development process of nursing professionals, and the related factors affecting professional commitment $\mathrm{Lu}$, et al. [3,5-7], there are also studies which explore the correlation between the value of work and pressure, and professional commitment $\mathrm{Ru}$, Lin, 2000; Chen, et al. [8]. The research of Wen et al. (2007) revealed that the level of satisfaction in work-study experience, and selfefficacy have a statistically significant and positive effect on professional commitment. The research of Liu, et al. [9] revealed that the completion of final courses can increase the level of professional commitment of nursing students. Still, there is a lack of profound discussion of other related factors' impact on nursing students' professional commitment, such as: willingness to exert effort, willingness for career involvement, positive professional assessment, and recognition of professional value. As such, this study analyzes the professional commitment of nursing students

in their final clinical electives from an empirical perspective, in order to understand the factors affecting this commitment and to improve the course quality of these final clinical electives. The goals of this study are as follows:

a) To explore the professional commitment status of nursing students who have completed their final six-week clinical electives in any medical department.

b) To explore the relationship between variables of the differing background of nursing students andprofessional commitment.

c) To understand the predictive power of variables of background for professional commitment and its component dimensions.

\section{Literature Review}

\section{The Connotations of "Professional Commitment"}

The sociologist Becker believes that commitment is the mechanism that reconciles humans' internal traits and behavior and thus the term is often used to as a conceptual description to delineate the characteristics of the actions of individuals or groups; as such, from a professional perspective, the term indicates the socialization of the professional role. Speaking generally, professional commitment refers to the attitude held by the individual toward their profession and is the nucleus and spirit that comprise a profession. On an individual level, the degree of stability in one's profession can be included in the concept, and overall, it makes it easy to achieve professional goals. Nursing professional commitment refers to the lifelong promise of nursing professionals that they are able to promote the development of the nursing profession, and that they maintain the will and act in order to improve the health and wellbeing of mankind Wang, et al. $[10,11]$. During their studies, nursing students are involved in both edifying and practical substantive work provided by professional nursing education; during this process, individuals' personal internalization is continuously stimulated, the socialization of the professional role is gradually incorporated, and loyalty and commitment to the nursing identity are formed. Overall, nursing professional commitment refers to the recognition of nurses, including: willingness to exert effort, willingness for career involvement, positive professional assessment, and recognition of professional value Li, et al. [12,13].

\section{Research Related to Nursing Professional Commitment}

In international research related to nursing professional commitment, personal variables related to nursing professional commitment are consolidated below. For gender, researchers have discovered that the professional commitment of females is higher than that of males (Ru, Lin, 2000). However, studies have also revealed that there are now differences between the nursing professional commitment of males and females Jie, Guo, Zheng, Hu, et al. [14]. For age, researchers have shown that those of greater age have higher levels of nursing professional commitment $\mathrm{Li}$, et al [15-17], while other researchers have pointed out that the level of nursing commitment is actually higher for those of lesser age Liu, et al. $[9,18,19]$. For schooling, some studies have shown thatnurses graduating from vocational and technical schools have higher levels of nursing professional commitment than that of higher education system graduates (Ru, Lin, 2000), while others have shown that the professional commitment of five-year junior college nursing students is higher than that of students studying at or above the university level Chen, et al.[2,20], and that different types of education system do not affect levels nursing professional commitment $\mathrm{Hu}$, et al. [1].

Considering motivation for studies, studies have revealed that students whose motivations were self-determination or personal interest had higher nursing professional commitment levels than students whose motivations were not self-determined Chen, et al. $[8,9,21]$. In view of family financial support, some studies indicate that the more satisfactory the economic situation, the higher the 
professional commitment of the nurse Jie et al., 2013; Wang, et al. $[20,22]$, still, some research has pointed out that a household's economic situation does not affect nursing professional commitment $\mathrm{Hu}$, et al. [1]). In considering personal perception of health, health covers both physiological and psychological health; the better the health status, the higher the level of professional commitment Wang, et al. [23,24]. Additionally, with the presence of depression, anxiety, or feelings of fear, professional commitment is lower Xie, et al. [13].Considering academic and work-study performance, those who scored above $80 \%$ in school and intheir work-study experience, willingness for nursing professional involvement was higher Chen, et al. [8], while another study indicated that differences in academic performance do not affect nursing professional commitment Liu, et al. [9].

Nursing professionals who work at different levels of a hospital were not shown to have different levels of professional commitment Wang, et al. [6]; Zhang, 2008; Liu, et al. [9]. Finally, in consideration of different medical departments, some studies have shown that nursing professionals working in the women \& children, and dialysis departments have higher levels of positive professional assessment and willingness for career involvement than those who work in intensive care, internal medicine, and surgical departments Wang, et al. [6]. Still, some studies have shown that there is no difference in the levels of professional commitment among nursing professionals working in different departments Cai, et al. [25].We can see from the above literature review that personal attribute factors related to the professional commitment of nursing professionals include: gender, age, schooling, academic motivation, family financial support, academic performance, work-study performance, level within the hospital, and medical department. These are included in the personal background variables for this study to explore the effect of varying personal background factors in the professionalcommitment of nursing students after completing their final six-week clinical elective courses.

\section{Research Methods}

\section{Research Subjects}

This study applied purposive sampling for its research subjects, namely nursing students at university in northern Taiwan who were in their final year of either two or four year technical programs in the nursing department during the 2012-2013 school year and who had completed their final clinical elective courses. It used a census to conduct a cross-sectional survey, distributing 402 questionnaires, and receiving 392 for a return rate of $97.51 \%$; after deducting those with incomplete responses, 389 valid questionnaires remained.

\section{Research Tools}

The research tools for this study include the two components of data on personal background, and a scale of nursing professional commitment. Data on personal background includes: gender, age, schooling, motivation for studying nursing, family financial support, personal perception of health, average grades in the previous academic semester, average grades in the previousworkstudy semester, hospital level of clinical electives, medical department of clinical electives,and outside work status. The scale of nursing professional commitment used here is that developed byLüGuiyun (2001), for which a letter of consent was obtained from the author. For this scale, validity checks and factor analysis were conducted by experts, and four dimensions of questions were extracted: willingness to exert professional effort (questions 1-16), willingness for professional career involvement (questions 17-24), positive professional career assessment (questions 25-29), and recognition of professional value (questions 30-34). For these 34 questions, a four-point Likert scale was provided, with responses: "extremely certain,," "certain,," "uncertain,," "extremely uncertain,," corresponding to scores of 4, 3,2, and 1 respectively, where higher points correspond to higher levels of professional commitment among nursing students. The Chrombach's $\alpha$ measuring the scale's internal consistency was valued at 0.93 , indicating good internal consistency.

\section{Data Collection Process}

Planning for this study went through the review of the Taipei City Hospital Institutional Review Board (IRB) (no. 1020216-E). Before the study commenced, research subjects were solicited; after obtaining the approval of the heads of relevant academic departments andprofessors, the goals of the study were explained to the research subjects. It was also explained that the method of research used anonymous completion of questionnaires, from which data would be encoded for presentation, the results of the survey would be provided only for academic research and not for other purposes. Also, that the study would completely respect the preservation of personal privacy and that the subjects can freely choose not to participate, or towithdraw from the study at any time. Before the study commenced, subjects' consent was obtained, and subjects signed consent forms.

\section{Results}

\section{Detailed Analysis of Nursing Student Types}

Among the subjects of this study, women were more prevalent. Students aged between 22.00-22.99 numbered 277 (71.21\%), the most common age group. For schooling, there were213 students in a two-year program (54.77\%), the most common schooling group. Students whose motivation for study was family desire totaled 95 $(24.42 \%)$, the most common motivation group. Students whose families provided total financial support numbered 291 (74.81\%), the most common financial support group. Students who perceived their health as good numbered 204 (52.44\%), comprising the most common group. Students whose average academic performance was between $80-84.9 \%$ numbered 214 (52.4\%), the most common 
group. Students whose average work-study performance was between 80-84.9 numbered 267 (68.64\%), comprising the most common group. Students whose elective course placed them in the medical center hospital level numbered 310 (79.69\%), comprising the most common group. Students whose elective courses belonged to the internal medicine department numbered 114 (29.31\%), comprising the most common group. Students whose outside work status was "none" numbered 346 (88.95\%), comprising the most common group; the average time spent engaged in outside work was 9.87 hours (Table 1 ).

Table 1: Personal Background Information.

\begin{tabular}{|c|c|c|c|c|c|c|c|c|c|}
\hline \multirow{2}{*}{ Personal Background } & \multicolumn{3}{|c|}{ Two-Year Program $(\mathrm{N}=\mathbf{2 1 3})$} & \multicolumn{3}{|c|}{ Four-Year Program $(\mathrm{N}=176)$} & \multicolumn{3}{|c|}{ Overall $(\mathrm{N}=389)$} \\
\hline & People & (\%) & $M(S D)$ & People & (\%) & $M(S D)$ & People & (\%) & $M(S D)$ \\
\hline \multicolumn{10}{|c|}{ Gender } \\
\hline Male & 2 & 0.9 & & 24 & 13.6 & & 26 & 6.7 & \\
\hline Female & 211 & 99.1 & & 152 & 86.4 & & 363 & 93.3 & \\
\hline \multicolumn{10}{|c|}{ Age } \\
\hline & & & $22.48(1.71)$ & & & $22.49(1.82)$ & & & $22.49(1.76)$ \\
\hline & & & $20.25-24.6$ & & & $20.25-25.25$ & & & $20-25.60$ \\
\hline \multicolumn{10}{|c|}{ Motivation for Study } \\
\hline Family Wishes & 37 & 17.4 & & 58 & 33 & & 51 & 15.1 & \\
\hline Decided by Test Scores & 30 & 14.1 & & 29 & 16.5 & & 50 & 14.8 & \\
\hline Ease of Employment & 36 & 16.9 & & 27 & 15.3 & & 64 & 19.0 & \\
\hline Personal Interest & 39 & 18.3 & & 28 & 15.9 & & 67 & 19.9 & \\
\hline Other & 24 & 11.3 & & 8 & 4.5 & & 104 & 31.2 & \\
\hline Two or More Motivations & 47 & 22.1 & & 26 & 14.8 & & 73 & 26.9 & \\
\hline \multicolumn{10}{|c|}{ Family Financial Support } \\
\hline Full Support & 152 & 71.4 & & 139 & 79 & & 291 & 74.8 & \\
\hline Only Tuition & 38 & 17.8 & & 27 & 15.3 & & 65 & 16.7 & \\
\hline No Support & 18 & 8.5 & & 8 & 4.5 & & 26 & 6.7 & \\
\hline Some Support & 5 & 2.3 & & 2 & 1.1 & & 7 & 1.8 & \\
\hline \multicolumn{10}{|c|}{ Perception of Personal Health } \\
\hline $\begin{array}{c}\text { Higher scores indicate worse } \\
\text { Health }\end{array}$ & 213 & & $2.39(0.84)$ & 176 & & $2.65(0.87)$ & 389 & & $2.52(0.86)$ \\
\hline \multicolumn{10}{|c|}{ Academic Performance } \\
\hline 85 and above & 93 & 43.7 & & 25 & 14.2 & & 118 & 30.3 & \\
\hline $80-84.9$ & 106 & 49.8 & & 96 & 54.5 & & 202 & 51.9 & \\
\hline $75-79.9$ & 13 & 6.1 & & 38 & 21.6 & & 51 & 13.1 & \\
\hline $70-74.9$ & 0 & 0 & & 16 & 9.1 & & 16 & 4.1 & \\
\hline 69.9 and below & 1 & 0.5 & & 1 & 0.6 & & 2 & 0.5 & \\
\hline \multicolumn{10}{|c|}{ Average Work-Study Performance } \\
\hline 85 and above & 58 & 27.2 & & 29 & 16.5 & & 87 & 22.4 & \\
\hline $80-84.9$ & 147 & 69 & & 120 & 68.2 & & 267 & 68.6 & \\
\hline $75-79.9$ & 7 & 3.3 & & 18 & 10.2 & & 25 & 6.4 & \\
\hline $70-74.9$ & & & & 9 & 5.1 & & 9 & 2.3 & \\
\hline
\end{tabular}




\begin{tabular}{|c|c|c|c|c|c|c|c|c|c|}
\hline 69.9 and below & 1 & 0.5 & & & & & 1 & 0.3 & \\
\hline \multicolumn{10}{|c|}{ Hospital Level } \\
\hline Medical Center & 170 & 79.8 & & 140 & 79.5 & & 310 & 79.7 & \\
\hline Regional Teaching & & & & & & & & & \\
\hline \multicolumn{10}{|l|}{ Hospital } \\
\hline & 38 & 17.8 & & 33 & 18.8 & & & & \\
\hline District Hospital & 5 & 2.3 & & 3 & 1.7 & & 8 & 2.1 & \\
\hline \multicolumn{10}{|c|}{ Medical Department of Elective } \\
\hline $\begin{array}{l}\text { Internal } \\
\text { Medicine }\end{array}$ & 61 & 28.6 & & 45 & 25.6 & & 106 & 27.2 & \\
\hline $\begin{array}{c}\text { Surgery, } \\
\text { Operating Room }\end{array}$ & 55 & 25.8 & & 40 & 22.7 & & 95 & 24.4 & \\
\hline Intensive Care & 57 & 26.8 & & 29 & 16.5 & & 86 & 22.1 & \\
\hline $\begin{array}{l}\text { Respiratory Care, Long-term } \\
\text { Care, Gynecological Cancer, } \\
\text { Rehab, Psychiatry }\end{array}$ & 9 & 4.2 & & 19 & 10.8 & & 28 & 7.2 & \\
\hline Other, Pediatrics & 31 & 14.6 & & 43 & 24.4 & & 74 & 19.0 & \\
\hline \multicolumn{10}{|c|}{ Outside Work Status } \\
\hline None & 185 & 86.9 & $10.20(6.16)$ & 161 & 91.5 & $9.27(4.53)$ & 346 & 88.9 & $9.87(5.61)$ \\
\hline Some & 28 & 13.1 & $2-28$ & 15 & 8.5 & $1.1-16$ & 43 & 11.1 & $1.1-28$ \\
\hline
\end{tabular}

Analysis of Professional Commitment after Completion of Final Electives

The average overall score for professional commitment of nursing students in this study was 96.08 , with a standard deviation (SD) of 15.03. A higher score indicates higher levels of professional commitment, with scores falling between 52 and 131. Within the questionnaire, the three items receiving the highest scores were, respectively: "Because nursing work helps others, it is very interesting" with a mean score (M) of 3.32, and an SD of 0.61 ; "I'm embarrassed to tell others that I study nursing (reverse-scoring question)" ( $\mathrm{M}=3.32, \mathrm{SD}=1.01)$; "Nursing work makes a clear contribution to the quality of human society" (M=3.21, $S D=0.64)$. The three items

receiving the lowest scores were, respectively: "I am willing to do nursing work for my entire life" (M=2.11, SD=0.81); "I would tell my friends that: 'nursing is a pretty ideal job."' $(M=2.43, \mathrm{SD}=0.78)$; "If in the future I were to undertake further study or training, I would change to a discipline other than nursing" ( $M=2.48, \mathrm{SD}=0.78)$.

This study discovered that for the four dimensions of professional commitment: "willingness to exert professional effort," "willingness for career involvement,," "positive career assessment,", and "recognition of professional value,," average scores for professional commitment were respectively 2.71, 2.93, 2.72, and 3.11. The overall level of professional commitment was 2.87. For the four dimensions of this scale and for the scale overall, mean scores were generally higher than the median (2.5).
After completing their final clinical electives, nursing students' professional commitment scores were in the mid-range level.

\section{Relationship Between Background Variables and Professional Commitment}

This study discovered that for "overall nursing professional commitment," nursing students in two-year programs had higher scores than those in four-year programs for the two dimensions of "willingness for career involvement," and "positive career assessment." Students whose motivation for study was "personal interest" and "ease of employment" had higher levels of professional commitment than those who responded "family desire" and "decided bytest-scores." For family financial support, students whose family financial support situation was "only provides school tuition and partial support" and "no support" had higher levels of nursing professional commitment than those who responded "full support." Students who had a good perception of their personal health had higher levels of professional commitment. For students whose academic performance was "85\% and above," "overall nursing professional commitment" and "willingness to exert professional effort" were higher than those whose performance was "80-84.9\%," and "79.9\% and below."

For nursing students whose work-study performance was " $85 \%$ and above," levels of "overall nursing professional commitment," "willingness to exert professional effort," "recognition of professional value," and "willingness for career involvement" were higher than those who scored "80-84.9\%" and "79.9\% and below." 
For students whose elective belonged to the "Medical Center," levels of "overall professional commitment," "willingness to exert professional effort," and "recognition of professional value" were all higher than students whose electives belonged to "regional teaching hospitals or district hospitals."Nursing students whose electives were in the "surgical ward, operating room," "internal medicinedepartment," and "emergency, intensive care ward" had higher levels of "willingness for careerinvolvement" compared to those in the "respiratory care ward, long-term care ward, gynecological cancer, rehabilitation department, or psychology department(Table 2).

Table 2: Relationship Analysis for Person Type and Professional Commitment(N=389).

\begin{tabular}{|c|c|c|c|c|c|c|c|c|c|c|c|}
\hline \multirow[t]{2}{*}{ Variable } & \multirow[t]{2}{*}{$\mathbf{N}$} & \multicolumn{2}{|c|}{$\begin{array}{l}\text { Overall Professional } \\
\text { Commitment }\end{array}$} & \multicolumn{2}{|c|}{$\begin{array}{c}\text { Willingness to Exert } \\
\text { Professional } \\
\text { Effort }\end{array}$} & \multicolumn{2}{|c|}{$\begin{array}{l}\text { Willingness for Career } \\
\text { Involvement }\end{array}$} & \multicolumn{2}{|c|}{$\begin{array}{c}\text { Positive Professional } \\
\text { Assessment }\end{array}$} & \multicolumn{2}{|c|}{$\begin{array}{l}\text { Recognition of Profes- } \\
\text { sional Value }\end{array}$} \\
\hline & & $M(S D)$ & $t / F / r$ & $M(S D)$ & $t / F / r$ & $M(S D)$ & $t / F / r$ & $M(S D)$ & $t / F / r$ & $M(S D)$ & $t / F / r$ \\
\hline Schooling ${ }^{\mathrm{a}}$ & & & $3.37 * *$ & & .99 & & $6.41^{* * *}$ & & $2.48^{*}$ & & .53 \\
\hline $\begin{array}{l}\text { (1) Two-year- } \\
\text { Program }\end{array}$ & 213 & 98.38(15.09) & & $43.86(8.63)$ & & $24.98(4.01)$ & & $13.92(2.93)$ & & $15.60(2.54)$ & \\
\hline $\begin{array}{l}\text { (2) Four-year- } \\
\text { Program }\end{array}$ & 176 & 93.28(14.51) & & $43.00(8.33)$ & & $21.47(6.27)$ & & $13.21(2.62)$ & & $15.47(2.40)$ & \\
\hline $\begin{array}{l}\text { Motivation } \\
\text { for Study }^{\mathrm{b}}\end{array}$ & & \multicolumn{2}{|c|}{$\begin{array}{c}11.76^{* * *} \\
\text { (3) }>\text { (1), (4) }>\text { (1), (4) }>\text { (2), (6)> (1) }\end{array}$} & & $\begin{array}{l}4.02 * * * \\
\text { (4) }>\text { (1) }\end{array}$ & \multicolumn{2}{|c|}{$\begin{array}{c}25.91 * * * \\
\text { (2) }>\text { (1), (3)> (1), (4)> (1), } \\
\text { (4)> (2), (5)> (1), (6)> (1) }\end{array}$} & \multicolumn{2}{|c|}{$\begin{array}{c}6.26 * * * \\
\text { (4) }>\text { (1), (6) }>\text { (1) }\end{array}$} & & $\begin{array}{l}5.06^{* * *} \\
\text { (4) }>\text { (1) }\end{array}$ \\
\hline $\begin{array}{l}\text { (1)Family } \\
\text { Wishes }\end{array}$ & 95 & 88.51(13.45) & & $41.87(7.74)$ & & $19.05(5.95)$ & & $12.47(2.68)$ & & $14.90(2.47)$ & \\
\hline $\begin{array}{l}\text { (2) Determined- } \\
\text { by Test Scores }\end{array}$ & 59 & $93.79(14.73)$ & & $42.89(10.2)$ & & $22.25(5.65)$ & & $13.25(2.67)$ & & $15.39(2.35)$ & \\
\hline $\begin{array}{l}\text { (3) Ease ofEm- } \\
\text { ployment }\end{array}$ & 63 & $96.31(12.26)$ & & $42.09(7.06)$ & & $25.09(3.16)$ & & $13.85(2.25)$ & & $15.27(2.20)$ & \\
\hline $\begin{array}{l}\text { (4) PersonalIn- } \\
\text { terest }\end{array}$ & 67 & $104.07(15.42)$ & & $46.77(8.82)$ & & $26.16(3.87)$ & & $14.62(2.98)$ & & $16.50(2.45)$ & \\
\hline (5) Other & 32 & $94.81(15.60)$ & & $41.59(8.58)$ & & $24.84(4.45)$ & & $13.50(2.98)$ & & $14.87(2.84)$ & \\
\hline $\begin{array}{l}\text { (6) Two or } \\
\text { More } \\
\text { Motivations }\end{array}$ & 73 & $100.75(13.83)$ & & $45.01(7.82)$ & & $25.34(4.05)$ & & $14.24(2.83)$ & & $16.15(2.34)$ & \\
\hline $\begin{array}{l}\text { Financial } \\
\text { Support }^{b}\end{array}$ & & & 2.62 & & .49 & $\begin{array}{r}6.81 \\
\text { (2) }>\text { (1), }\end{array}$ & & & $3.03^{* *}$ & & 1.03 \\
\hline $\begin{array}{l}\text { (1) Full } \\
\text { Support }\end{array}$ & 295 & $95.10(14.57)$ & & $43.31(8.26)$ & & $22.84(5.63)$ & & $13.40(2.80)$ & & $15.47(2.44)$ & \\
\hline $\begin{array}{l}\text { (2) Tuition Only } \\
\text { and Partial- } \\
\text { Support }\end{array}$ & 68 & $99.47(16.41)$ & & $44.38(9.34)$ & & $24.92(4.47)$ & & $14.23(2.83)$ & & $15.92(2.50)$ & \\
\hline $\begin{array}{c}\text { (3) No } \\
\text { Support }\end{array}$ & 26 & 98.15(15.50) & & $42.92(9.05)$ & & $25.73(4.21)$ & & $14.19(2.69)$ & & $15.30(2.79)$ & \\
\hline $\begin{array}{l}\text { Perception } \\
\text { of Personal } \\
\text { Health }^{c}\end{array}$ & 389 & $96.07(15.03)$ & $-.180^{* * *}$ & $43.47(8.50)$ & $4.52^{* *}$ & $23.39(5.44)$ & $-.25^{* * *}$ & $13.60(2.81)$ & -.08 & $15.54(2.48)$ & -.08 \\
\hline $\begin{array}{c}\text { Academic } \\
\text { Performance }^{\mathrm{b}}\end{array}$ & & $\begin{array}{r}5.65 \\
\text { (1) }>\text { (2), }\end{array}$ & & $\begin{array}{r}5.88 \\
\text { (1) }>\text { (2), (1) }\end{array}$ & & & 2.40 & & .66 & & 2.18 \\
\hline
\end{tabular}




\begin{tabular}{|c|c|c|c|c|c|c|c|c|c|c|c|}
\hline (1) 85 分个 & 120 & $99.69(16.26)$ & & 45.54(9.39) & & $24.28(5.36)$ & & 13.85(2.98) & & $15.85(2.78)$ & \\
\hline (2) $80-84.9$ & 214 & 94.92(13.95) & & 42.84(7.77) & & 23.07(5.46) & & $13.49(2.74)$ & & $15.50(2.18)$ & \\
\hline (3) $79.9 \downarrow$ & 55 & 92.65(15.06) & & 41.41(8.41) & & $22.72(5.40)$ & & $13.49(2.76)$ & & $15.01(2.80)$ & \\
\hline $\begin{array}{c}\text { Work-Study } \\
\text { Performance }^{\mathrm{b}}\end{array}$ & & \multicolumn{2}{|c|}{$\begin{array}{c}7.20^{* * *} \\
\text { (1) }>\text { (2), (1) > (3) }\end{array}$} & \multicolumn{2}{|c|}{$\begin{array}{c}\mathbf{7 . 0 8}^{* * *} \\
\text { (1) }>\text { (2), (1) > (3) }\end{array}$} & & $\begin{array}{l}4.54 * \\
\text { (1) }>\text { (3) }\end{array}$ & & 2.14 & & $\begin{array}{l}4.47 * \\
\text { (1) }>\text { (2) }\end{array}$ \\
\hline (1) $85 \uparrow$ & 84 & $100.92(15.86)$ & & $46.39(8.72)$ & & $24.26(5.56)$ & & $14.03(3.03)$ & & $16.23(2.55)$ & \\
\hline (2) $80-84.9$ & 263 & 95.31(14.31) & & $42.87(8.14)$ & & $23.47(5.18)$ & & $13.57(2.74)$ & & $15.39(2.36)$ & \\
\hline 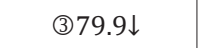 & 42 & 91.14(15.56) & & 41.38(9.03) & & 21.21(6.28) & & $12.95(2.77)$ & & $15.11(2.81)$ & \\
\hline $\begin{array}{c}\text { Hospital } \\
\text { Level }^{\mathrm{a}}\end{array}$ & & & $1.99 *$ & & $2.31^{*}$ & & -.34 & & 1.42 & & $2.04 *$ \\
\hline $\begin{array}{l}\text { (1) Medical } \\
\text { Center }\end{array}$ & 310 & 96.83(14.69) & & $44.04(8.00)$ & & $23.35(5.60)$ & & $13.70(2.80)$ & & $15.67(2.42)$ & \\
\hline $\begin{array}{l}\text { (2) Regional } \\
\text { Teaching } \\
\text { Hospital } \\
\text { and District } \\
\text { Hospital }\end{array}$ & 79 & $93.07(16.03)$ & & $41.25(9.96)$ & & $23.58(4.79)$ & & $13.20(2.87)$ & & $15.03(2.63)$ & \\
\hline $\begin{array}{c}\text { Medical } \\
\begin{array}{c}\text { Department } \\
\text { of } \\
\text { Elective }^{b}\end{array}\end{array}$ & & & .53 & & 1.13 & $\begin{array}{l}5.69 * * * \\
\text { (1) }>\text { (4), } \\
\text { (2) }>\text { (4), } \\
\text { (3) }>\text { (4) }\end{array}$ & & & .75 & & 1.08 \\
\hline $\begin{array}{l}\text { (1) Internal } \\
\text { Medicine }\end{array}$ & 106 & 96.01(15.94) & & $42.55(9.34)$ & & $24.32(4.41)$ & & $13.64(2.89)$ & & $15.31(2.73)$ & \\
\hline $\begin{array}{l}\text { (2) Surgery, } \\
\text { Operating } \\
\text { Room }\end{array}$ & 95 & $96.69(14.24)$ & & $43.25(8.06)$ & & $24.21(4.22)$ & & $13.83(2.49)$ & & $15.40(2.43)$ & \\
\hline $\begin{array}{l}\text { (3) Intensive- } \\
\text { Care }\end{array}$ & 86 & $95.67(15.25)$ & & $43.15(8.10)$ & & 23.47(5.37) & & $13.55(3.02)$ & & $15.48(2.44)$ & \\
\hline $\begin{array}{l}\text { (4) Respiratory } \\
\text { Care, } \\
\text { Long-term } \\
\text { Care, Gyneco- } \\
\text { logical Cancer, } \\
\text { Rehab, } \\
\text { Psychiatry }\end{array}$ & 28 & 92.53(11.78) & & $44.28(7.45)$ & & $19.53(7.23)$ & & $12.78(2.60)$ & & $15.92(2.14)$ & \\
\hline (5) Other & 74 & $97.16(15.64)$ & & $45.14(8.55)$ & & $22.40(6.69)$ & & $13.62(2.94)$ & & $15.98(2.30)$ & \\
\hline
\end{tabular}

Note: ${ }^{*} \mathrm{p}<.05 .{ }^{* *} \mathrm{p}<.01 .{ }^{* * *} \mathrm{p}<.001$; Statistical test method: a: Independent sample t test; $\quad \mathrm{b}$ : One-way ANOVA (If F-test shows significance, Scheffe's method for post-hoc analysis is used) ; c: Person's correlation

Stepwise Regression Analysis of Personal Background Variables

Using stepwise regression analysis, the variance inflation factor (VIF) for personal background variables on nursing professional commitment overall and in each dimension was calculated for each predictive variable, which all fell between 1.00-1.02. Regression coefficient tolerance values were all between 0.97-0.99, and the residuals (using the Durbin-Watson Test) were between 1.201.95 , near to the ideal value of 2 . This shows compliance with non-multilinear basic assumptions. From Table 3, we can see that the predictive variables proved to be statistically significant with respect to the regression coefficients for "overall professional commitment;" these were: academic performance $(\mathrm{t}=-4.10, \mathrm{p}<$ 0.001), perception of personal health $(t=-3.81, p<0.001)$, family financial support $(\mathrm{t}=2.94, \mathrm{p}<0.01)$, and motivation for study $(\mathrm{t}=$ $2.33, \mathrm{p}<0.05)$.For the dimension "willingness to exert professional effort," variables which reached statistical significance were: motivation for study $(\mathrm{t}=2.57, \mathrm{p}<0.01)$, perception of personal health $(\mathrm{t}=-4.79, \mathrm{p}<0.001)$, academic performance $(\mathrm{t}=-3.75, \mathrm{p}$ $<0.001)$, and medical department of clinicalelective $(\mathrm{t}=2.57, \mathrm{p}<$ $0.05)$. 
Table 3: Analysis of Research Subject Professional Commitment N=389.

\begin{tabular}{|c|c|c|c|c|c|}
\hline Type (No. of Questions) & Highest Value & Lowest Value & Average Value & Standard Deviation & Rank \\
\hline Overall Level of Nursing Professional Commitment (34) & 131 & 52 & 2.87 & 0.78 & \\
\hline Willingness to Exert Professional Effort (16) & 64 & 17 & 2.71 & 0.74 & 4 \\
\hline Willingness for Career Involvement (8) & 32 & 8 & 2.93 & 0.89 & 2 \\
\hline Positive Career Assessment (5) & 20 & 6 & 2.72 & 0.84 & 3 \\
\hline Recognition of Professional Value (5) & 20 & 9 & 3.11 & 0.65 & 1 \\
\hline
\end{tabular}

For the dimension "willingness for career involvement," significant variables were: school system ( $t=-6.09, \mathrm{p}<0.001)$, family financial support $(\mathrm{t}=2.73, \mathrm{p}<0.05)$, and medical department of elective $(\mathrm{t}=-2.91, \mathrm{p}<0.01)$. For the dimension "positive professional assessment," variables that achieved statistical significance were: family financial support $(t=2.56, p<0.05)$, perception of personal health $(\mathrm{t}=-2.66, \mathrm{p}<0.01)$, and academic performance $(\mathrm{t}=-2.13$, $\mathrm{p}$ $<0.05)$. For "recognition of professional value," variables achieving statistical significance were: motivation for study $(\mathrm{t}=3.20, \mathrm{p}<$ $0.001)$, academic performance $(t=-2.23, p=0.05)$, and hospital level $(\mathrm{t}=-2.07, \mathrm{p}<0.05)$. The $\mathrm{F}$ values for overall significance tests for the overall regression model were, respectively: $12.37,13.63$, 19.87, 6.01, 7.45 (all $p$ values were: $p<0.001$ ).

\section{Discussion}

\section{Current Status of Professional Commitment}

The results of this study reveal that for "professional commitment" overall, and for each dimension, values fell between 2.71 and 3.11. Of the four dimensions, the score for "recognition of professional value" was highest. For overall professional commitment, the average score for each question was 2.87 , a midlevel score. From this we can see that nursing students who have completed their final clinical electives have a positive perception of overall professional commitment, which is similar to the findings of Lü, et al. [5,1,8], that nursing students have a mid-level of professional commitment. For the subjects of this study, who have completed their final clinical electives, scores for "recognition of professional value" were highest, followed by "willingness for career involvement," "positive career assessment," and "willingness to exert professional effort"; these results are consistent with related Taiwanese studies which have also found that recognition of professional value received the highest scores, and willingness to exert professional effort the lowest Wang, et al., [5,6,8,20]. So, nursing students recognize the value of the nursing profession, but are less willing to exert effort in carrying out nursing work.

Primarily, this could be due to a poor work environment, low wages, frustration, severe work pressure, few opportunities for advancement, not being respected, and an increase in medical disputes Chen, et al. $[8,26]$. Additionally, due to the high turnover rate of nurses in the current working environment, the workload of nurses is excessively large (Zhou, 2012); this causes nursing students to have a low willingness to exert professional effort.The results of this study found that the three items measuring nursing professional commitment were, in descending order: "Because nursing work helps others, it is very interesting," "I'm embarrassed to tell others that I study nursing (reverse-scoring question)," and "Nursing work makes a clear contribution to the quality of human society"; this reveals that nursing students approve of the work done by nurses after graduation Lü, et al.[5,27]. The three items measuring nursing professional commitment that received the lowest scores were, in ascending order: "I am willing to do nursing work for my entire life," "I would tell my friends that: 'nursing is a pretty ideal job.," and "If in the future I were to undertake further study or training, I would change to a discipline other than nursing."; this could be due to the fact that clinical work pressure exceeds individuals' physical and mental capacity, causing a decrease in the willingness of nursing students to exert effort (Cai et al., 2012).

The results of this study reveal that with regard to schooling systems, nursing students in two-year programs have higher overall professional commitment, "willingness for career involvement," and "positive career assessment" than students in four-year programs; this is consistent with other Taiwanese studies Chen, et al. [8,23,20]. Furthermore, Chen, et al. [8] and Cai et al. (2001) have pointed out that different school systems do not affect "willingness to exert professional effort," "positive career assessment," or "recognition of professional value"; this is similar to the results of this study, and is because students in two-year programs undergo five years of specialized study, then take on advanced training, causing their relatively high recognition of professional commitment. Differingmotivations for study had a statistically significant effect on scores for overall professional commitment and each of its component facets; this result is consistent with the findings of Chen, et al. $[8,9,21]$. For students whose motivation for study is personal interest, the higher the positive assessment, the higher the level of nursing professional commitment.Family financial support did not prove to have significant effects on "willingness for career involvement" or "positive professional assessment," we propose that this may be due to the fact that the subjects of the study were nursing students, and those who were not, or only partially supported bore responsibility for their finances; thus, these students had a relatively high willingness for career involvement. For perception of personal health, those students who perceived 
their health to be "very good" and "good" had higher levels of willingness to exert professional effort and positive professional assessment than those students who perceived their health as "poor" and "very poor."

Another Taiwanese study pointed out that nurses with tough personalities and who perceive their health as good have higher levels of professional commitment (Han, 2007); this is consistent with the results of this study.For variation in the academic performance of nursing students, overall, and for "willingness to exert professional effort," grades had a statistically significant relationship with professional commitment; a Taiwanese study showed that nursing students whose academic grades were higher than $80 \%$ had higher levels of professional commitment than those students who scored below 79\% Chen, et al. [8], a result consistent with the results of this study. Still, Chen, et al. [8] further proposed that the higher the grades of a nursing student, the greater their professional commitment dimension "willingness for career involvement." The results of this study show that the higher a nursing student's grades, the higher the professional commitment dimension "willingness to exert professional effort," inconsistent with Chen's results. Our result could be because nursing students with higher academic results have higher expectations for their results, and are more willing to make attempts Huang, et al. [28]. Considering variation in work-study performance, this variable reached the significant level for professional commitment overall, and for the dimensions of "willingness to exert professional effort," "recognition of professional value," and "willingness for professional involvement."

Other Taiwanese studies have revealed that previous grades are not significantly related with nursing professional commitment Liu, et al. [9], which is inconsistent with the findings of this study. Still, a study has shown that students whose grades were $80 \%$ and higher had higher levels ofnursing professional commitments when compared with students whose grades were $79 \%$ and below Chen, et al. [8].For students, electives in different hospital levels were shown to have significantly effects on overall professional commitment, as well as the dimensions "willingness to exert professional effort," and "recognition of professional value;" this shows that the level of nursing professional commitment for nursing students in the "medical center" was higher than those in "regional teaching hospitals and district hospitals." A study by Chen et al. (2010) showed that the hospital level of nursing student electives does not affect levels of nursing professional commitment; these results are inconsistent with our own, and we propose that our results may be due to the fact that the degree of care that nursing students in the medical center experience is high, which causes them to have a correspondingly higher willingness to exert effort Liao [29]. For nursing students whose electives belong to different medical departments, the dimension "willingness for career involvement" was shown to be significantly affected. Those students with electives in "internal medicine," "surgery and operating room," and "emergency and intensive care unit" had higher levels of professional commitment, which we propose may be due to the fact that the working abilities of students in these departments improved rapidly, causing a rise in their selfconfidence towards their nursing work Ye, et al. [30]; Lin, Zhang, Chen, Cai, Huang, 2000.A Taiwanese study has pointed out that nurses in dialysis rooms and women and children departments have higher levels of professional commitment than those in internal medicine and surgery Wang, et al. [6], while another study shows that there is no significant difference in the levels of nursing professional commitment between nurses working in different medical departments Liu, et al. [9]; the results of these studies are inconsistent with our own results.

\section{Predictive Factors for Professional Commitment}

Through its stepwise regression analysis, this study discovered that the four variables of academic performance, perception of personal health, family financial support, and motivation for study can predict overall professional commitment. This shows that for nursing students with better grades and better perception of personal health, no family financial support, and who are motivated by personal interest or ease of employment can be predicted to have better professional commitment (Table 4). Further, academic performance, perception of personal health, family financial support, motivation for study, and medical department of elective are all predictive variables for "willingness to exert professional effort." This shows that nursing students whose elective belongs to the chronic long-term care ward can be predicted to have better willingness toexert professional effort. School-system, family financial support, and medical department of elective are predictive variables for "willingness for career involvement."

Table 4: Stepwise Regression Analysis for Personal Characteristics and Professional Commitment.

\begin{tabular}{|c|c|c|c|c|c|c|}
\hline \multirow{2}{*}{ Variable Name } & \multicolumn{3}{|c|}{ Willingness to Exert Professional Effort } & \multicolumn{3}{|c|}{ Willingness for Career Involvement } \\
\hline & $\beta(\mathrm{SE})$ & t-value & VIF & $\beta(\mathrm{SE})$ & t-value & VIF \\
\hline Schooling & & & & $-3.20(.52)$ & $-6.09^{* * *}$ & 1.01 \\
\hline $\begin{array}{c}\text { Family Financial } \\
\text { Support }\end{array}$ & & & & $.90(.38)$ & $2.37^{*}$ & 1.01 \\
\hline $\begin{array}{l}\text { Motivation for } \\
\text { Study }\end{array}$ & $.36(.14)$ & $2.57^{* *}$ & 1.01 & & & \\
\hline
\end{tabular}




\begin{tabular}{|c|c|c|c|c|c|c|}
\hline $\begin{array}{l}\text { Perception of } \\
\text { Personal Health }\end{array}$ & $-2.26(.47)$ & $-4.79 * * *$ & 1.01 & & & \\
\hline $\begin{array}{l}\text { Academic } \\
\text { Performance }\end{array}$ & $-1.92(.51)$ & $-3.75^{* * *}$ & 1.01 & & & \\
\hline $\begin{array}{c}\text { Medical } \\
\text { Department of } \\
\text { Elective }\end{array}$ & $.60(.28)$ & $2.11^{*}$ & 1.01 & $-.53(.18)$ & $-2.91^{* *}$ & 1.01 \\
\hline F-Value & \multicolumn{3}{|c|}{$13.633^{* * *}$} & \multicolumn{3}{|c|}{$19.872^{* * *}$} \\
\hline Adjusted $\mathrm{R}^{2}$ & \multicolumn{3}{|c|}{.115} & \multicolumn{3}{|c|}{.127} \\
\hline \multirow{2}{*}{ Variable Name } & \multicolumn{3}{|c|}{ Positive Professional Assessment } & \multicolumn{3}{|c|}{ Recognition of Professional Value } \\
\hline & $\beta(\mathrm{SE})$ & t-value & VIF & $\beta(\mathrm{SE})$ & t-value & VIF \\
\hline $\begin{array}{l}\text { Family Financial } \\
\text { Support }\end{array}$ & $53(.20)$ & $2.56^{*}$ & 1.00 & & & \\
\hline $\begin{array}{l}\text { Perception of } \\
\text { Personal Health }\end{array}$ & $-.43(.16)$ & $-2.66^{* *}$ & 1.01 & & & \\
\hline $\begin{array}{l}\text { Motivation for } \\
\text { Study }\end{array}$ & & & & $.14(.04)$ & $3.20^{* * *}$ & 1.00 \\
\hline $\begin{array}{c}\text { Academic } \\
\text { Performance }\end{array}$ & $-.37(.18)$ & $-2.13^{*}$ & 1.01 & $-.35(.16)$ & $-2.23^{*}$ & 1.02 \\
\hline Hospital Level & & & & $-.55(.27)$ & $-2.07^{*}$ & 1.02 \\
\hline F-Value & \multicolumn{3}{|c|}{$6.013^{* * *}$} & \multicolumn{3}{|c|}{$7.452^{* * *}$} \\
\hline Adjusted $\mathrm{R}^{2}$ & \multicolumn{3}{|c|}{.037} & \multicolumn{3}{|c|}{.048} \\
\hline \multirow{2}{*}{ Variable Name } & \multicolumn{6}{|c|}{ Overall Professional Commitment } \\
\hline & $\beta(\mathrm{SE})$ & t-value & VIF & & & \\
\hline Academic Performance & $-3.71(.91)$ & $4.10^{* * *}$ & 1.01 & & & \\
\hline Perception of Personal Health & $3.21(.84)$ & $-3.81^{* * *}$ & 1.01 & & & \\
\hline Family Financial Support & $3.10(1.06)$ & $2.94^{* *}$ & 1.01 & & & \\
\hline F-Value & \multicolumn{3}{|c|}{$12.368^{* * *}$} & & & \\
\hline Adjusted $\mathrm{R}^{2}$ & \multicolumn{3}{|c|}{.105} & & & \\
\hline
\end{tabular}

This shows that students in two-year programs, those in internal medicine, surgery, and in the intensive care unit can be predicted to have better "willingness for career involvement." Predictive variables for "positive professional assessment were academic performance, perception of personal health, and family financial support. For "recognition of professional value," predictive variables included motivation for study, academic performance, and hospital level; this shows that for students whose final clinical electives belong to the medical center, better "recognition of professional value" can be predicted. This is largely in line with the results of Lü et al. (1998), Lu, et al. [7,5,8], with regard to the motivation for study, school system, hospital level, and academic performance of nursing students[31-34].

\section{Conclusion}

a) The average level of professional commitment for nursing students who have completed their final clinical electives was 2.87, a mid-level score. Considering each dimension of commitment, scores for "recognition of professional value" were highest.

b) For variables of different backgrounds and professional commitment, eight variables caused statistically significant variation. Two-year school system, motivation by personal interest or ease of employment, no or only partial family financial support, and better perception of personal help all resulted in better professional commitment. In addition, academic and work-study grades of $85 \%$ and above, electives in the medical center, and those in internal medicine, intensive care, and operating rooms were also shown to have better professional commitment.

c) Considering the predictive value of variables for different personal backgrounds for professional commitment, the best predictive variables were academic performance, perception of personal health, family financial support, motivation for study, medical department of elective, school system, and hospital level.

\section{Suggestions}

The results of this study reveal that as academic and workstudy performance increase, so to do the overall and component dimensions of professional commitment. Thus, this study recommends that nursing students be assisted in their studies to achieve positive academic success, which will increase their professional commitment. Additionally, electives in internal 
medicine, surgery, and intensive care yielded higher willingness for career involvement than other departments. For this reason, we suggest that final clinical electives offer opportunities for nursing students to improve their working skills; this will improve the self-confidence of students in their work. This study, like many others, shows that of all dimensions of professional commitment, willingness to exert professional effort received the lowest score. Thus, this study suggests that when nursing students return to school, professors use this time to address problems and conflicts encountered in their final clinical electives, provide appropriate counseling, and clarify doubts and flawed conceptions of their future nursing work. At the same time, we suggest that the government request medical institutions to implement reasonable nurse-patient ratios to avoid over-working clinical nurses. This over-work causes fatigue, poor work environments, and reduces the willingness of nursing students to enter the profession.

\section{Limitations}

This study faced manpower, material, and time constraints, and used only the nursing students of the nursing program at a northern Taiwan university as its subjects and scope. Thus, its results cannot yield comprehensive conclusions. In order to improve the sense of mission of graduated nursing students, we recommend that future studies expand the scope and subjects of research, and understand the influencing factors for the professional commitment of students who have completed their final clinical electives. At the same time, future research can move towards longitudinal studies to better understand how the professional commitment of nursing students varies at different points in time during their final clinical electives, as well as factors which influence these differences.

\section{References}

1. Hu HY, Chen TY, Chiu SI (2009) The simple Explored on Effect of Professional Commitment Caused by Knowledge management Ability, and Self-determined Motivation of Nursing Students. Leadership Nursing 10(2): 44-56.

2. Wu HM, Liu PF, Ho HH, Chen PL, Chao HL, et al. (2012) Reducing Occupational Burnout and Enhancing Job Performance in New Nurses: The Efficacy of "Last Mile" Programs .Journal of Nursing 59(4): 30-42.

3. Lu KY, Chen YC, Wang MC (1998) Analysis of the Nursing Competence of Senior Nursing Students. Med Education 2(2): 180-189.

4. Benlıgıray S, Sönmez H (2011) Relationships of Nurses' Professional Commitment with other Commitment Forms: Organizational Commitment, Work Commitment and Family Commitment. Hacettepe University Faculty of Health Sciences Nursing Journal 18(1): 28-40.

5. Lu KY, Chang YY, Chiou SL (2001) Changes in Nursing Professional Commitment Among Junior College Graduates. The Journal of Nursing Research 9(1): 28-38.

6. Wang CL, Lu KY, Huang CY, Ho MY (2008) Professional Commitment and Its Related Factors in Nursing Staff. Chang Gung Nursing 19(3): 334-345.

7. Lu MS, Lin CF (2000) Work Value and Professional Commitment among New Graduates of Nursing Schools. Formosan J Med 4(2): 115-126.

8. Chen NW, Chang TH, Yeh MC (2012) Correlations of Work Value and Professional Commitment among Nursing Students. Cheng Ching Medical Journal 8(1): 15-25.
9. Liu HY, Chang CC, Yu KL, Ho YC, Tzeng YJ, et al. (2010) The Implementation and Learning Effects of Last-Mile Curriculum among Nursing Students of a Four-Year Technology College. Journal of Research in Education Sciences 55(4): 131-155

10. Wang CL, Tsai LY, Lu KY (2005) A Conceptual Analysis of Professional Commitment. Journal of Nursing 52(5): 71-77.

11. Ohlen J, Segesten K (1998) The professional identity of the nurse: concept analysis and development. Journal of Advanced Nursing 28(4): 720-727.

12. Lee HI, Lee S (2002) Literature Review of Professional Commitment and Career Development in Nursing. Tzu Chi Nursing Journal 1(2): 7-11.

13. Xie J, Wang HC (2008) Investigation undergraduate nursing professional commitment and mental health. Chinese J Modern Nurs 14(10): 11931194.

14. Kou YC, Hsieh MR, Cheng CC, Hu HY (2013) Correlations between Nurses' Job Satisfaction, Professional Commitment, and Organizational Commitment. Leadership Nursing 14(2): 22-32.

15. Lee SF, Ma CC, Chiou CP, Su SF (2012) The Professional Commitment of Nurses in a Public Psychiatric Teaching Hospital. Journal of Nursing and Healthcare Research 8(2): 83-93.

16. Li RH, Chang FM, Lai HL, Lee RP (2008) A Study of the Professional Commitment and Work Values among Public Health Nurses in Taitung County, Taiwan. Tzu Chi Nursing Journal 7(2): 79-92.

17. Lin PL, Ho MY, Tzeng LC, Lu KY (2005) Work Stress and Professional Commitment Among Nurses in Community Health Centers. Journal of Evidence-Based Nursing 1(2): 132-139.

18. Waugaman WR, Lohrer DJ (2000) From nurse to nurse anesthetist: The influence of age and gender on professional socialization and career commitment of advanced practice nurses Original Research Article. Journal of Professional Nursing 16(1): 47-56.

19. Teng CI (2008) Moderating Effects of Professional Commitment in the Relationship between Turnover Intention and Factors of Job Satisfaction. Chang Gung Nursing 19(1): 24-40.

20. Wang KY, Chou CC, Huang JL (2010) A Study of Work Values, Professional Commitment, Turnover Intention and Related Factors Among Clinical Nurses. Journal of Nursing 57(1): 22-34.

21. Nesje K (2015) Nursing students' prosocial motivation: does it predict professional commitment and involvement in the job? Journal of Advanced Nursing 71(1): 115-125.

22. Tsai HC, Huang LC, Liu SC, Wang HC, Li TC, et al. (2007) Nurses' Professional Commitment and Job Satisfaction. Mid-Taiwan Journal of Medicine 12(2): 100-108.

23. Wang WW, Li CF (2012) Mediating effect of nursing interns' professional commitment between carry decision-making self-efficacy and mental health. Journal of Nursing Administration 12(8): 541-543.

24. Lin B (2006) A Study on Commitment to Major career self-efficacy and mental health of the Higher Vocational Students and Their Relationship. Chengdu College of Education 20(9).

25. Tsai LY, Chiang MC, Li YC, Lu KY (2001) Professional Commitment of Nurses at District Hospitals in KSH Areas. Chang Gung Nursing 12(4): 294-304.

26. Yeh MC (2007) Expecting Job Stress and Resources Needed by Graduating Nursing Students Pre-entry to Nursing Occupation. Studies in the Humanities and Social Science 51: 37-50.

27. Lu KY, Chiou SL (1998) Professional Commitment of Nursing Students. The Journal of Nursing Research 6(2): 109-120.

28. Huang IC, Chen YJ, Huang PW (2003) The Influence of Academic Achievement on Effort Attribution-The Example of Students in the Food \& Beverage Department from 2-Year Colleges implementing the 
Sandwich Style Instruction. Commerce \& Management Quarterly 4(2): 149-161.

29. Liao HY (2011) Relationship between the professional commitments and caring behaviors among the in-job nursing students -- An example from the University of Technology in central Taiwan. Taichung: Central Taiwan University of Science and Technology.

30. Yeh SC, Liu CH (2009) A Study of Medical-Surgical Nursing Competency for Nursing College Graduates. Journal of Cardinal Tien College of Nursing 7: 43-45.

31. Chou SM (2012) Response of Taiwan Nursing Education to Today's Nursing Shortage. Journal of Nursing 59(5): 24-29.

ISSN: 2574-1241

DOI: 10.26717/BJSTR.2020.30.004966

Shu-Chen Wang. Biomed J Sci \& Tech Res

(C) (i) This work is licensed under Creative

Submission Link: https://biomedres.us/submit-manuscript.php
32. HUI MH (2007) The Investigation of the Relationship among Personality Hardiness Uncertainty and Nursing Professional Commitment. Taipei: Chang Gung University of Nursing.

33. Lin YC, Chang M, Chen ZT, Tsai LY, Hwang SL (2009) Evaluation of Nursing Education and Curriculum by Baccalaureate Nursing Graduates Working in the Hospital. Journal of Medical Education 4(3): 315-328.149-161.

34. Den JF, Chang HC (2007) The Impacts of Internship Circumstance in Sandwich Curriculum on Students' Professional Commitment: the Case of Taiwan's International Hotels. Journal of Human Resource Management 7(2): 49-70.

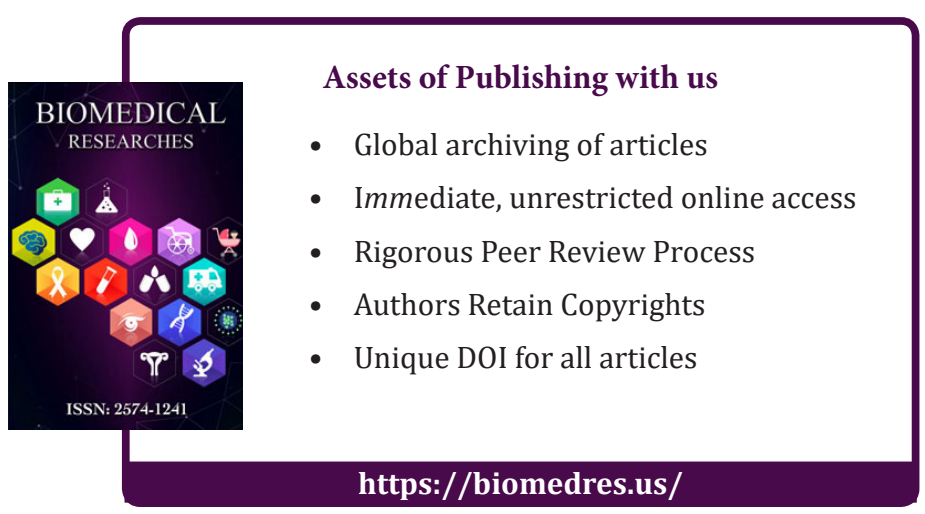

